\title{
Line-transitive, point-imprimitive linear spaces: the grid case
}

\author{
Anton Betten* \\ Gregory Cresp \\ Cheryl E. Praeger
}

full screen

close

\section{Abstract}

For a fixed integer $k$, all but a finite number of line-transitive linear spaces with lines of size $k$ are point-primitive. In this paper, we study the finite class of examples where a line-transitive group is point-imprimitive, that is, preserves a non-trivial partition of the point set. We restrict to the case where (i) the number of unordered point-pairs, on a given line, contained in the same class of the partition is at most eight, and (ii) some nonidentity group element fixes setwise each class of the partition, and also fixes a point. This family of linear spaces was studied by Ngo Dac Tuan and the third author in 2003, leaving several problems unresolved. We prove that all examples in this family are known, namely Desarguesian projective planes of appropriate orders, and an additional example on 91 points. The result is obtained by a combination of theoretical analysis, and exhaustive computer search.

Keywords: linear space, block design, finite projective plane, line-transitive, pointimprimitive

MSC 2000: 05B05, 05B25, 20B25

\section{Introduction}

A finite linear space $\mathcal{S}=(\mathcal{P}, \mathcal{L})$ consists of a finite set $\mathcal{P}$ of points, and a set $\mathcal{L}=\left\{\lambda_{1}, \ldots, \lambda_{b}\right\}$ of subsets of $\mathcal{P}$, called lines, such that each pair of points lies in a unique line, and each line contains at least two points. The automorphism

\footnotetext{
*The authors acknowledge a grant of computing time from the Australian Partnership for Advanced Computing (APAC). This research forms part of the Australian Research Council project DP0557587. The third author acknowledges support of an Australian Research Council Federation Fellowship.
} 


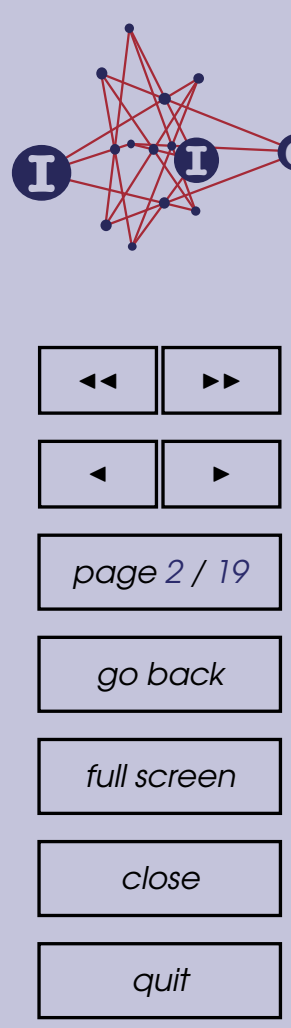

group $\operatorname{Aut}(\mathcal{S})$ of $\mathcal{S}$ consists of all permutations of $\mathcal{P}$ that leave $\mathcal{L}$ invariant, and $\mathcal{S}$ is called line-transitive if $\operatorname{Aut}(\mathcal{S})$ is transitive on $\mathcal{L}$. More generally, we say that a linear space $\mathcal{S}$ has a group theoretic property if some subgroup of $\operatorname{Aut}(\mathcal{S})$ has the property. In particular, for a line-transitive linear space $\mathcal{S}$, all lines have the same size $k$ say, and hence $\mathcal{S}$ is a $2-(v, k, 1)$ design with $v=|\mathcal{P}|$ the number of points of the linear space.

The major result that inspired this investigation is due to Delandtsheer and Doyen [8] (see Theorem 1.1) and shows that, in a sense, line-transitive linear spaces are almost always point-primitive. That is, for a given line-size, there is only a finite number of linear spaces which are line-transitive but not point-primitive. Put in a different way, linear spaces with these properties are counterexamples to the (wrong) assertion that line-transitivity implies pointprimitivity. The result of Delandtsheer and Doyen shows that these examples are exceptional and hence deserve attention. In this paper, we study these exceptional examples of line-transitive point-imprimitive linear spaces.

Let us describe the history of the study of these counterexamples briefly. The theorem of Delandtsheer and Doyen shows that if a line-transitive group of automorphisms of a linear space $\mathcal{S}=(\mathcal{P}, \mathcal{L})$ with line-size $k$ leaves invariant a non-trivial partition of $\mathcal{P}$, then $|\mathcal{P}| \leq\left(\left(\begin{array}{l}k \\ 2\end{array}\right)-1\right)^{2}$. It was shown in [3, 12] that this upper bound could not be sharp if $k \neq 8$, and in [11] that there are exactly 467 pairwise non-isomorphic linear spaces that attain the bound when $k=8$.

Line-transitive, point-imprimitive linear spaces were investigated further in [13] in the special case where (i) the number of inner pairs of a given line, that is, the number of unordered point-pairs of the line that lie together in the same class of an invariant partition, is at most eight, and (ii) some non-identity automorphism fixes setwise each class of the partition, and also fixes a point. In this situation, Praeger and Tuan [13, Theorem 1.6] showed that there is only a small number of feasible parameter sets.

Our aim in this paper is to complete this classification by dealing with a rather difficult case, namely case (c) of [13, Theorem 1.6], about which not much could be said until now. In Theorem 1.2 below, we show that this case does indeed lead to examples, but that all these examples are known.

We remark that parallel to this study, a massive investigation of the general case for line-transitive, point-imprimitive linear spaces has been undertaken. It is general in the sense that the number of inner pairs on a line is no longer restricted to be small, and there are no restrictions on the classwise stabiliser. That study is reported in [2], and is independent of this work. It relies only on [13, Theorem 1.6], not on Theorem 1.2 below. Similarly, the classification achieved in this paper does not follow from the classification in [2] as our classification assumes no bound on $k / \operatorname{gcd}(k, v)$. 


\subsection{Line-transitive, point-imprimitive linear spaces}

A partition $\mathcal{C}$ of a finite set $\mathcal{P}$ is a set of pairwise disjoint subsets whose union equals the set $\mathcal{P}$. The subsets $C \in \mathcal{C}$ are called parts or classes of $\mathcal{C}$. The partition $\mathcal{C}$ is called trivial if either $\mathcal{C}$ consists of only one class or $\mathcal{C}$ contains only oneelement classes, otherwise $\mathcal{C}$ is said to be non-trivial. A $\mathcal{C}$-inner pair (or simply an inner pair, if the partition $\mathcal{C}$ is clear from the context) is an unordered pair of points $\alpha, \beta \in \mathcal{P}$ which belong to the same class of $\mathcal{C}$. Otherwise, if the points belong to two different classes of $\mathcal{C}$, we say that they form a $\mathcal{C}$-outer pair.

Let $G$ be a group that acts on a set $\mathcal{P}$. Then $G$ is said to leave a partition $\mathcal{C}$ invariant if, for all $g \in G$ and all $C \in \mathcal{C}$, the image $C^{g}$ is also a class of $\mathcal{C}$. In particular, $\mathcal{C}$-inner pairs are mapped to $\mathcal{C}$-inner pairs under elements of $G$. Let $G$ be transitive on $\mathcal{P}$. If there exists a non-trivial $G$-invariant partition of $\mathcal{P}$ then $G$ is said to be imprimitive on $\mathcal{P}$. Otherwise, $G$ is primitive on $\mathcal{P}$.

We remark that in studying a group $G$ of automorphisms of a linear space $\mathcal{S}=(\mathcal{P}, \mathcal{L})$, the group may not coincide with $\operatorname{Aut}(\mathcal{S})$, the full automorphism group of $\mathcal{S}$. Also, a linear space is said to be trivial if it has only one line, or if all its lines have only two points; otherwise it is called non-trivial. Thus $\mathcal{S}$ is non-trivial if $2<k<|\mathcal{P}|$.

The result from [8] mentioned above, and stated as Theorem 1.1 below, shows among other things that, for a linear space admitting a line-transitive, point-imprimitive automorphism group, the number of points is bounded above by a function of the line size. This means that for a given line size $k$ there is only a finite number of line-transitive, point-imprimitive linear spaces.

Theorem 1.1 (Delandtsheer-Doyen parameters [8]). Let $\mathcal{S}=(\mathcal{P}, \mathcal{L})$ be a nontrivial linear space admitting a line-transitive automorphism group $G$ that leaves invariant a non-trivial partition $\mathcal{C}$ of $\mathcal{P}$ with $d$ classes of size $c$. Let $x$ be the number of $\mathcal{C}$-inner pairs of a line, and let $k$ be the line size. Then there exists another positive integer $y$ such that

$$
c=\frac{\left(\begin{array}{l}
k \\
2
\end{array}\right)-x}{y} \text { and } d=\frac{\left(\begin{array}{l}
k \\
2
\end{array}\right)-y}{x} .
$$

We call the pair $(x, y)$ the Delandtsheer-Doyen parameters corresponding to $\mathcal{C}$. By [13, Theorem 1.1(a)],

$$
x=\frac{\left(\begin{array}{l}
k \\
2
\end{array}\right)(c-1)}{c d-1} \quad \text { and } \quad y=\frac{\left(\begin{array}{l}
k \\
2
\end{array}\right)(d-1)}{c d-1},
$$

and hence the integer triples $(c, d, k)$ and $(x, y, k)$ mutually determine each other. Moreover, if $(c, d, k)$ corresponds to $(x, y, k)$ then $(d, c, k)$ has $(y, x, k)$ as 


\begin{abstract}
$\frac{1}{1} 0$
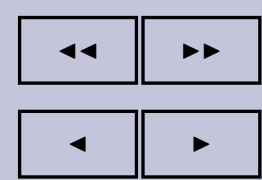

page $4 / 19$

go back

full screen

its mate, though in general there will not exist a $G$-invariant partition of $\mathcal{P}$ with $c$ classes of size $d$. Recent attempts, for example in [4], have aimed at classifying line-transitive, point-imprimitive linear spaces for small values of $k$. This article is a step towards a classification in the case where the number $x$ of inner pairs on a line is small. We remark further that while the first of the DelandtsheerDoyen parameters $x$ has a combinatorial interpretation as the number of inner pairs on a line, no similar meaning is known for the second parameter $y$ in general. However, there is an interpretation for $y$ when $G$ preserves a grid structure on $\mathcal{P}$. We will explore this in section 3.
\end{abstract}

close

quit

ACADEMIA

PRESS

WW

$\underline{\underline{11 \|}}$

UNIVERSITEIT

GENT

\subsection{The main result}

For a transitive group $G$ on a set $\mathcal{P}$ and a $G$-invariant partition $\mathcal{C}=\left\{C_{1}, \ldots, C_{d}\right\}$ of $\mathcal{P}$, the kernel of $G$ on $\mathcal{C}$ is the subgroup $G_{(\mathcal{C})}$ of elements $g \in G$ with $C_{i}^{g}=C_{i}$ for $i=1, \ldots, d$. We say that $\mathcal{C}$ is $G$-normal if $G_{(\mathcal{C})}$ is transitive on each of the $\mathcal{C}$-classes.

In [13], Praeger and Tuan examined line-transitive point-imprimitive linear spaces where the first Delandtsheer-Doyen parameter is small and the partition is normal. The current work aims at resolving the open cases in part (c) of their Theorem 1.6, thereby proving the result promised in the remarks following the statement of [13, Theorem 1.6]. Part (a) of [13, Theorem 1.6] addresses the situation where the only group element fixing a point, and fixing each class of the partition setwise, is the identity element. Parts (b) and (c) (which contain the unresolved cases) address the opposite situation, namely where there is a non-identity element that fixes a point and fixes each class setwise. Note that we do not include as an assumption that the partition is normal. Instead we prove this as a consequence of the existence of this element. Our result is as follows. Its proof will be presented in section 4.

Theorem 1.2. Let $\mathcal{S}=(\mathcal{P}, \mathcal{L})$ be a non-trivial linear space admitting a linetransitive group $G$ that leaves invariant a non-trivial point-partition $\mathcal{C}$. Assume further that the number of $\mathcal{C}$-inner pairs on a line is at most 8 , and that some non-trivial element of $G$ fixes each $\mathcal{C}$-class setwise and also fixes at least one point. Then either

(i) $\mathcal{S}$ is a Desarguesian projective plane of order 4, 9 or 16, or

(ii) $\mathcal{S}$ is the Colbourn-McCalla design constructed in [6] with 91 points and line size 6.

Moreover, each of these linear spaces has a line-transitive, point-imprimitive subgroup of automorphisms satisfying the hypotheses above. 


\section{Some parameters for line-transitive, point-im- primitive linear spaces}

\subsection{Base lines}

Let $G$ act on a set of points $\mathcal{P}$. For a nonnegative integer $s$, we denote the set of $s$-subsets of $\mathcal{P}$ by $\mathcal{P}^{\{s\}}$. For $S \subseteq \mathcal{P}$, let $S^{G}$ denote the $G$-orbit of $S$, that is, the set of images $S^{g}$ for all elements $g$ in $G$, and let $G_{S}$ denote the setwise stabiliser of $S$ in $G$. Under certain conditions, the $G$-orbit of a $k$-subset $\lambda \subseteq \mathcal{P}$ can be taken as the set of lines of a linear space admitting $G$ as a line-transitive automorphism group. In this case, we say that $\lambda$ is a base line for the $G$-linetransitive linear space $\left(\mathcal{P}, \lambda^{G}\right)$. We will characterize base lines shortly. First, we examine some properties of $G$-orbits on pairs. In a linear space the unique line containing two points $\alpha$ and $\beta$ is denoted by $\lambda(\alpha, \beta)$. Recall that the number of lines of a linear space is generally denoted by $b$ and equals $\left(\begin{array}{l}v \\ 2\end{array}\right) /\left(\begin{array}{l}k \\ 2\end{array}\right)$, where $v=|\mathcal{P}|$ and $k$ is the line size.

Lemma 2.1. Let $G$ be a line-transitive automorphism group of a linear space $\mathcal{S}=(\mathcal{P}, \mathcal{L})$ with line size $k$ and $|\mathcal{L}|=b$. Then the length of every G-orbit $\mathcal{O}$ on $\mathcal{P}^{\{2\}}$ is divisible by $b$. In particular, the number of orbits of $G$ on $\mathcal{P}^{\{2\}}$ is at most $\left(\begin{array}{l}k \\ 2\end{array}\right)$.

Proof. For every pair of points $\{\alpha, \beta\}, G_{\{\alpha, \beta\}}$ fixes $\lambda(\alpha, \beta)$, and hence $G_{\{\alpha, \beta\}} \leq$ $G_{\lambda(\alpha, \beta)}$. Thus $b=\left|\lambda(\alpha, \beta)^{G}\right|=\left|G: G_{\lambda(\alpha, \beta)}\right|$ divides $\left|G: G_{\{\alpha, \beta\}}\right|=\left|\{\alpha, \beta\}^{G}\right|$. In particular $|\mathcal{O}| / b$ is a positive integer, where $\mathcal{O}:=\{\alpha, \beta\}^{G}$. Counting all pairs of points yields the upper bound on the number of those orbits: $\left(\begin{array}{l}v \\ 2\end{array}\right)=$ $b \cdot \sum_{\mathcal{O}}|\mathcal{O}| / b \geq\left(\left(\begin{array}{l}v \\ 2\end{array}\right) /\left(\begin{array}{l}k \\ 2\end{array}\right)\right) \cdot \sum_{\mathcal{O}} 1$, and hence $\left(\begin{array}{l}k \\ 2\end{array}\right) \geq \sum_{\mathcal{O}} 1$.

We call a transitive permutation group $G$ on $\mathcal{P}$ feasible (for a particular linear space parameter set) if the length of every orbit on $\mathcal{P}^{\{2\}}$ is divisible by $b$. Lemma 2.2 gives a criterion for testing whether a $k$-subset $\lambda$ is a base line for a $G$-line-transitive linear space. The criterion involves the integers $\mu(\mathcal{O}, S)$ defined in (3), for $G$-orbits $\mathcal{O}$ on $\mathcal{P}^{\{2\}}$ and $k$-subsets $S$ of $\mathcal{P}$.

$$
\mu(\mathcal{O}, S)=\left|\mathcal{O} \cap S^{\{2\}}\right|=|\{\{\gamma, \delta\} \in \mathcal{O}:\{\gamma, \delta\} \subseteq S\}|
$$

Note that $\mu(\mathcal{O}, S)$ does not depend on the choice of $S$ within its $G$-orbit $S^{G}=$ $\left\{S^{g} \mid g \in G\right\}$.

Lemma 2.2 (Orbit Lemma). Let $G$ be a group acting on a set $\mathcal{P}$ of $v$ points. Let $k$ be a positive integer greater than 2 such that $b=\left(\begin{array}{l}v \\ 2\end{array}\right) /\left(\begin{array}{l}k \\ 2\end{array}\right)$ is an integer. Then $\lambda \in \mathcal{P}^{\{k\}}$ is a base line of a linear space with point set $\mathcal{P}$ if and only if both 


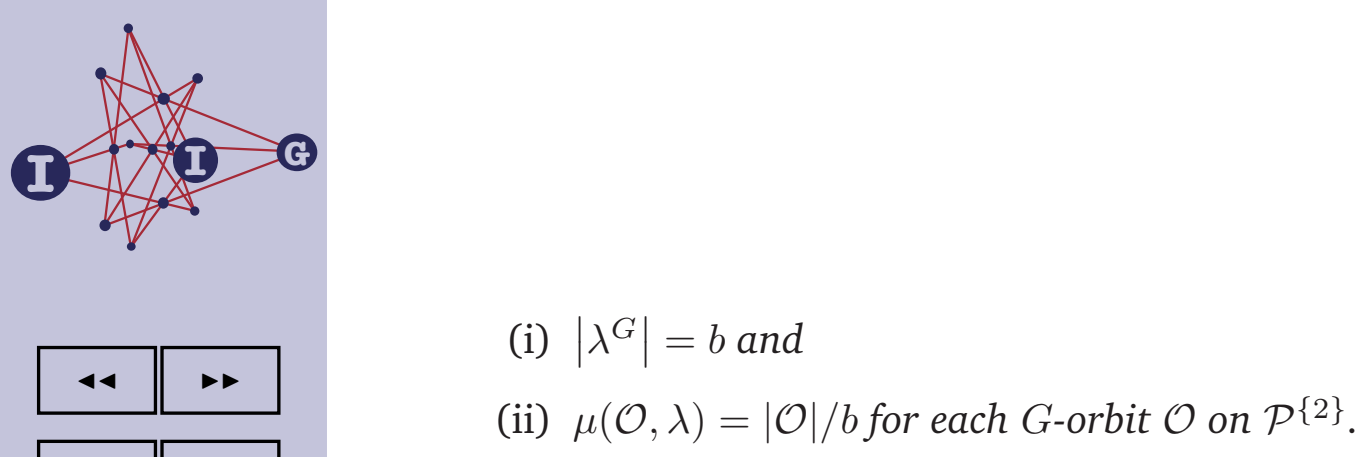

A proof can be found, for example, in [3, Proposition 1.3]: one shows that $\mu(\mathcal{O}, \lambda) /|\mathcal{O}|$ is a constant, say $a$, independent of the $G$-orbit $\mathcal{O}$ in $\mathcal{P}^{\{2\}}$, if and only if each pair of points lies in a constant number of the $k$-subsets in $\lambda^{G}$. An easy counting argument then shows that this number is $a\left|\lambda^{G}\right|$. Thus $\lambda$ is a base line for a linear space if and only if the constant $a$ is $1 / b$ where $b=\left|\lambda^{G}\right|$, that is, if and only if (i) and (ii) both hold.

We mention some elementary facts about automorphisms of linear spaces.

Lemma 2.3. Let $G$ be a group of automorphisms of a linear space $\mathcal{S}=(\mathcal{P}, \mathcal{L})$, and let $\lambda \in \mathcal{L}, A \subset \mathcal{P}$, and $g \in G$.

(a) If $A \subseteq \lambda$ and $|A| \geq 2$, then $G_{A} \leq G_{\lambda}$.

(b) The image $\lambda^{g}=\lambda$ if and only if $\lambda$ is a union of $\langle g\rangle$-orbits.

(c) If the order $|g|$ of $g$ is 2 then $g$ fixes some line setwise.

Proof. For part (a), let $\alpha, \beta$ be distinct points of $A$. Since $\lambda$ is the unique line containing $\{\alpha, \beta\}$ it follows that $\lambda$ is the unique line containing $A$. Therefore $G_{A} \leq G_{\lambda}$. Part (b) is obvious. For part (c), let $|g|=2$ and let $\alpha, \beta$ be distinct points interchanged by $g$. Then $g$ fixes $\{\alpha, \beta\}$ setwise and hence by part (a), $g$ fixes setwise the line $\lambda(\alpha, \beta)$.

\subsection{Intersection numbers}

Let $\mathcal{S}=(\mathcal{P}, \mathcal{L})$ be a line-transitive point-imprimitive linear space with respect to a group $G$ of automorphisms and a non-trivial partition $\mathcal{C}$ with $d$ classes of size $c$, and let $\lambda \in \mathcal{L}$. Then the intersection numbers defined as

$$
d_{i}=|\{C \in \mathcal{C}:|C \cap \lambda|=i\}|,
$$

for $0 \leq i \leq k$ are independent of the choice of the line $\lambda$ and satisfy

$$
\sum_{i=1}^{k} d_{i}\left(\begin{array}{l}
i \\
2
\end{array}\right)=x .
$$

The intersection type is the vector $\left(1^{d_{1}}, \ldots, k^{d_{k}}\right)$, where we usually omit components $i^{d_{i}}$ if $d_{i}=0$. The spectrum is the set of non-zero intersection numbers

$$
\operatorname{spec} \mathcal{S}:=\left\{i>0 \mid d_{i} \neq 0\right\} \text {. }
$$

We sometimes write $\operatorname{spec}_{\mathcal{C}} \mathcal{S}$ if we need to specify the partition $\mathcal{C}$. 


\section{Grid structures on points}

Throughout this section, $\mathcal{S}=(\mathcal{P}, \mathcal{L})$ denotes a linear space that admits a linetransitive group $G$ leaving invariant a non-trivial partition $\mathcal{C}=\left\{C_{1}, \ldots, C_{d}\right\}$ of the point set $\mathcal{P}$ with $d$ classes of size $c$. Sometimes $G$ leaves invariant a second partition $\mathcal{D}$ such that $|C \cap D|=1$ for all $C \in \mathcal{C}$ and $D \in \mathcal{D}$. In this case $\mathcal{D}=\left\{D_{1}, \ldots, D_{c}\right\}$ with each $\left|D_{j}\right|=d$ and every point $\alpha$ of $\mathcal{P}$ may be labelled by the unique ordered pair of integers $(i, j)$ such that $\{\alpha\}=C_{i} \cap D_{j}$. We write $\alpha=\alpha_{i, j}$. Thus we can parametrize the points by the Cartesian product

$$
\{1, \ldots, d\} \times\{1, \ldots, c\}
$$

such that

$$
\mathcal{P}=\left\{\alpha_{i, j} \mid 1 \leq i \leq d, \quad 1 \leq j \leq c\right\} .
$$

The classes of the partitions $\mathcal{C}$ and $\mathcal{D}$ can then be thought of as the rows and columns respectively of the matrix

$$
\left(\begin{array}{cccc}
\alpha_{1,1} & \alpha_{1,2} & \cdots & \alpha_{1, c} \\
\alpha_{2,1} & \alpha_{2,2} & \cdots & \alpha_{2, c} \\
\vdots & & & \vdots \\
\alpha_{d, 1} & \alpha_{d, 2} & \cdots & \alpha_{d, c}
\end{array}\right),
$$

that is,

$$
C_{i}=\left\{\alpha_{i, j} \mid 1 \leq j \leq c\right\}, \quad 1 \leq i \leq d
$$

and

$$
D_{j}=\left\{\alpha_{i, j} \mid 1 \leq i \leq d\right\}, \quad 1 \leq j \leq c .
$$

We call such a set $\mathcal{P}$ together with a distinguished ordered pair of partitions $(\mathcal{C}, \mathcal{D})$ as above a grid of type $d \times c$ and write $\mathcal{P}=\mathcal{G}_{d \times c}^{(\mathcal{C}, \mathcal{D})}$. We call $\mathcal{C}$ the rowpartition and $\mathcal{D}$ the column-partition of the grid. Furthermore, the intersection types of a line with respect to the partitions $\mathcal{C}$ and $\mathcal{D}$ are called the row intersection type and column intersection type, respectively.

Desarguesian projective planes for which the number $v$ of points is not a prime power provide line-transitive examples: write $v=d \cdot c$ with $d$ and $c$ coprime, and take $G$ to be a Singer group, that is, a cyclic group $\langle g\rangle$ of automorphisms that is regular on points (and hence also on lines). Then the orbit sets of $\left\langle g^{d}\right\rangle$ and $\left\langle g^{c}\right\rangle$ on points are $G$-invariant point-partitions $\mathcal{C}$ and $\mathcal{D}$ that form a $\operatorname{grid} \mathcal{G}_{d \times c}^{(\mathcal{C}, \mathcal{D})}$.

A grid is said to be trivial if at least one of the numbers $c$ or $d$ is 1 , that is, if $\mathcal{C}$ and $\mathcal{D}$ are trivial partitions. We also say that $G$ preserves a grid if $G$ preserves a pair of non-trivial partitions that forms a grid. In fact if $G$ preserves 






\begin{tabular}{|c|c|c|c|c|c|c|c|c|c|c|c|}
\hline \multirow[b]{2}{*}{44} & \multirow[b]{2}{*}{$\rightarrow$} & line & $k$ & $c^{\prime}$ & $d^{\prime}$ & $x^{\prime}$ & $y^{\prime}$ & $d_{1}^{\prime}$ & $d_{2}^{\prime}$ & $b^{(r)}$ & comment \\
\hline & & 1 & 10 & 7 & 13 & 3 & 6 & 4 & 3 & 1 & \multirow[t]{6}{*}{ projective plane } \\
\hline \multirow[b]{2}{*}{4} & \multirow[b]{2}{*}{ 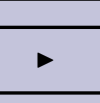 } & 2 & 10 & 41 & 11 & 4 & 1 & 2 & 4 & 5 & \\
\hline & & 3 & 11 & 17 & 13 & 4 & 3 & 3 & 4 & 2 & \\
\hline \multirow{2}{*}{\multicolumn{2}{|c|}{ page $13 / 19$}} & 4 & 12 & 61 & 13 & 5 & 1 & 2 & 5 & 6 & \\
\hline & & 5 & 16 & 113 & 17 & 7 & 1 & 2 & 7 & 8 & \\
\hline \multirow{2}{*}{\multicolumn{2}{|c|}{ go back }} & 6 & 17 & 43 & 19 & 7 & 3 & 3 & 7 & 3 & \\
\hline & & 7 & 17 & 13 & 21 & 6 & 10 & 5 & 6 & 1 & projective plane \\
\hline
\end{tabular}

full screen

close

Table 1: Parameters for Lemma 4.1

corresponds to case 14 analysed on page 60 of [13]. It is proved there that in all of these cases, $K \simeq D_{2 c^{\prime}}$, and $X$ is defined as $C_{G}(K)$, the kernel of the natural conjugation action of $G$ on $K$. It is observed that $X \cap K=1$ and $G / X \leq \operatorname{Aut}(K)$. However it is wrongly asserted that $\operatorname{Aut}(K) \simeq Z_{c^{\prime}} \cdot Z_{c^{\prime}-1}$. In fact $\operatorname{Aut}(K) \simeq K \simeq D_{2 c^{\prime}}$ since $c^{\prime}$ is odd. Now $X \cap K=1$ implies that $K \simeq X K / X \leq G / X \leq \operatorname{Aut}(K) \simeq K$, and hence $G=X K \simeq X \times K$ in all these cases. Moreover, since an involution in $K$ fixes a line of $\mathcal{S}$, it follows that $b$ divides $|G| / 2$. In lines 2-6, $|G| / 2=c^{\prime} d^{\prime} h=v h$, and hence $b^{(r)}$ divides $h$. This divisibility condition also holds for line 1 since in that case $b^{(r)}=1$. This completes the proof.

From now on we will assume that $\mathcal{S}$ is neither $\mathrm{PG}_{2}(4)$ nor the ColbournMcCalla design. Note that Lemmas 3.2 and 3.3 apply to all lines of Table 1 , proving in particular that $G$ preserves a grid structure on the point set.

Corollary 4.2. In each of lines 1-7 of Table $1, G$ preserves a grid structure $\mathcal{P}=$ $\mathcal{G}_{d^{\prime} \times c^{\prime}}^{\left(\mathcal{C}^{\prime}, \mathcal{D}\right)}$ on points, where $\mathcal{D}$ is the set of $X$-orbits in $\mathcal{P}$ with $X$ as in Lemma 4.1. Moreover, the $X$-action on $\mathcal{C}^{\prime}$ is equivalent to its action on each of these orbits.

Proof. Suppose that one of the lines of Table 1 holds. Then all the conditions of Lemma 3.2 hold relative to the partition $\mathcal{C}^{\prime}$, and hence $G$ preserves a second point-partition $\mathcal{D}$ such that $\mathcal{P}=\mathcal{G}_{d^{\prime} \times c^{\prime}}^{\left(\mathcal{C}^{\prime}, \mathcal{D}\right)}$ is a grid of type $d^{\prime} \times c^{\prime}$. Moreover, each part $D$ of $\mathcal{D}$ is the fixed point set of an involution $u$ in $K=G_{\left(\mathcal{C}^{\prime}\right)}$. Now $\langle u\rangle$ is a point stabiliser in $G_{\left(\mathcal{C}^{\prime}\right)}$, and its normaliser in $G$ is $X \times\langle u\rangle$. Therefore $X \times\langle u\rangle$ acts transitively on the fixed point set $D$ of $\langle u\rangle$, and thus $D$ is an $X$-orbit. Hence $\mathcal{D}$ is the set of $X$-orbits in $\mathcal{P}$. Clearly the $X$-action on $\mathcal{C}^{\prime}$ is equivalent to its action on each of these orbits.

Our next step is to prove that lines 2-6 do not lead to any examples.

Lemma 4.3. There are no examples for lines 2-6 of Table 1. Moreover, in the case of line $1, G=X \times G_{(\mathcal{C})}$, where $X \simeq Z_{13}$ and $G_{(\mathcal{C})}=D_{14}$. 

We may therefore assume that each non-trivial, intransitive normal subgroup of $X$ has 3 orbits of length 7 in $D$. In this case $M$ acts faithfully on each orbit of degree 7 . Suppose that $M \neq Z_{7}$. Then, for all possibilities, $M$ has trivial centre and $\operatorname{Aut}(M) / M \leq Z_{2}$. Thus $\left|X /\left(M C_{X}(M)\right)\right|$ is not divisible by 3 , and so $C_{X}(M)$ permutes transitively the three $M$-orbits in $D$ and $C_{X}(M) \cap M=1$. This time, if $a \in M$ has order 7 , and $z \in C_{X}(M)$ has order 3 , then $\langle a z\rangle \simeq Z_{21}$ and is regular on $D$. Finally suppose that $M=Z_{7}$. If $C_{X}(M)$ is transitive on $D$ then we obtain a subgroup $Z_{21}$ of $C_{X}(M)$ acting regularly as required, while if $C_{X}(M)$ is intransitive on $D$ then $C_{X}(M)=M$ and $X \leq \mathrm{AGL}(1,7)$. In this last case we have a subgroup $F_{21}$ of $X$ acting regularly on $\bar{D}$.

We complete consideration of lines 1 and 7 in the following section.

\subsection{The projective plane cases}

Lemma 4.5. The only projective plane of order 9 admitting a line-transitive action of the cyclic group $Z_{91}$ is the Desarguesian plane $\mathrm{PG}_{2}(9)$. Moreover $\mathrm{PG}_{2}(9)$ admits a line-transitive group $G \cong Z_{13} \times D_{14}$ corresponding to line 1 of Table 1 .

Proof. Suppose that $\mathcal{S}=(\mathcal{P}, \mathcal{L})$ is a projective plane of order 9 admitting a line-transitive cyclic subgroup $G=\langle a\rangle \cong Z_{91}$ of automorphisms. Note that the point set $\mathcal{P}$ can be identified with the set $G$ in such a way that $G$ acts by multiplication. The $G$-action is line-transitive and point imprimitive, preserving the point partition $\mathcal{C}=\left\{C_{i} \mid 0 \leq i<13\right\}$, where $C_{i}=\left\{a^{13 j+i} \mid 0 \leq j<7\right\}$. Thus the algorithm in [1] may be used to find all such projective planes up to isomorphism. A computer search using this algorithm led to 12 base lines of (possibly isomorphic) projective planes. The search for the designs took $20 \mathrm{sec}-$ onds on a Macintosh PowerBook G4, during which around 3 million backtrack nodes were examined by the program. Using the software package nauty [10] (version 2.2), the 12 designs from the search were all identified as the Desarguesian projective plane $\mathrm{PG}_{2}(9)$. This proves the first assertion.

Finally we consider $\mathcal{S}=\mathrm{PG}_{2}(9)$. The normaliser of a Singer cycle in $\mathrm{P} \Gamma \mathrm{L}(3,9)$ is $Z_{91}: Z_{6}$ and contains a unique subgroup of the form $Z_{91}: Z_{2}$. This subgroup may be identified with a subgroup $Z_{91}$ of the multiplicative group of a field of order $3^{6}$, extended by a field automorphism of order 2 that fixes pointwise a subfield of order $3^{3}$. Thus this subgroup is isomorphic to $Z_{13} \times D_{14}$ (as in Lemma 4.3) and so $\mathrm{PG}_{2}(9)$ is the unique linear space corresponding to line 1 of Table 1.

Lemma 4.6. Up to isomorphism, there is a unique linear space corresponding to line 7 of Table 1, namely the Desarguesian projective plane $\mathrm{PG}_{2}(16)$. 



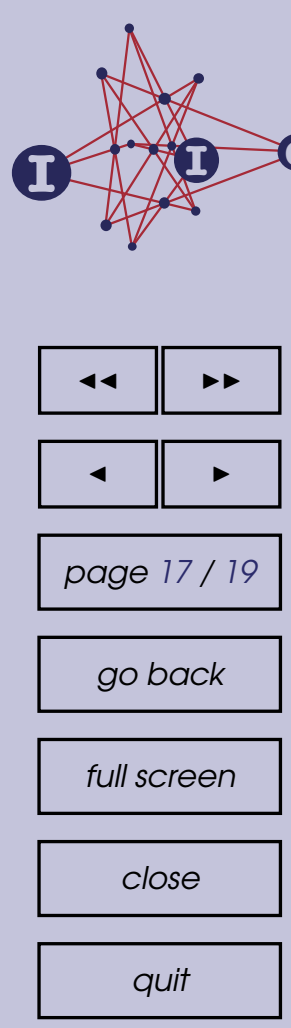

$\alpha_{2}=\alpha_{0,1}$. Thus we began with the set $A=\left\{\alpha_{1}, \alpha_{2}\right\}$ (a horizontal pair), and we used the fact that $G_{\lambda}$ contains the involution $s$ that fixes this pair. During the search we made sure that we chose whole orbits under $\langle s\rangle$ for addition to the current set. We did not re-compute the setwise stabiliser $G_{A}$ during the procedure when $A$ was enlarged. This was because we did not have a fast algorithm for computing set stabilisers. The extra information that might have been gained from a possibly larger group, was offset by the time that would have been used for this computation.

For the first group with $G_{(\mathcal{D})}=Z_{21}$, the search on a Macintosh PowerBook G4 quickly yielded 12 base lines. The computing time was less than 2 minutes. A total number of around 8.3 million backtrack nodes were examined by the algorithm. It turned out that all linear spaces generated by the 12 base lines were isomorphic to the Desarguesian projective plane $\mathrm{PG}_{2}(16)$. This was verified by computing for each design the canonical form of its incidence matrix using the software package nauty [10]. Here, a canonical form of the incidence matrix of a design is simply a unique representative of all incidence matrices of designs isomorphic to the given design. Once the canonical form has been computed, isomorph checking is equivalent to equality testing of the corresponding canonical incidence matrices. In order to prove that the designs were isomorphic to $\mathrm{PG}(2,16)$, the latter design was constructed separately and its canonical incidence matrix was computed as well. It turned out that the canonical incidence matrices of the 12 designs and the canonical incidence matrix of $P G(2,16)$ were all identical. This proves that the designs are all isomorphic to $P G(2,16)$. In order to treat incidence matrices of designs with nauty, a graph had to be created which encodes the design. This graph is a bipartite graph, with vertices corresponding to points and blocks of the design. The edges in the graph correspond to incident point/line pairs. The isomorphism types of projective planes correspond to the isomorphism classes of this type of graph, where the isomorphisms are required to preserve the bipartition. In order to have nauty compute the canonical form with respect to this class of isomorphisms, the bipartition was handed to nauty in the form of an initial partition of the vertices of the graph. The details of how to specify such a partition are described in the nauty User Guide [10].

For the second group with $G_{(\mathcal{D})}=F_{21}$, the computer found no solutions at all. The search took less than one minute time. A total number of around 3.6 million backtrack nodes were examined by the algorithm. The best that could be found were 4620 partial baselines of size 13 . This completes the proof. 


\section{References}

[1] A. Betten, G. Cresp, A. C. Niemeyer and C. E. Praeger, A search algorithm for line-transitive, point-imprimitive linear spaces, Bayreuth. Math. Schr. 74 (2005), 42-57.

[2] A. Betten, A. Delandtsheer, M. Law, A. C. Niemeyer, C. E. Praeger and S. Zhou, Finite line-transitive linear spaces: theory and search strategies, preprint, available at http://arxiv.org/abs/math.C0/0701629v2 (2008).

[3] P. J. Cameron and C. E. Praeger, Block-transitive $t$-designs. I. Pointimprimitive designs, Discrete Math. 118(1-3) (1993), 33-43.

[4] A. R. Camina and S. Mischke, Line-transitive automorphism groups of linear spaces, Electron. J. Combin. 3(1) (1996), Research Paper 3, approx. $16 \mathrm{pp}$. (electronic).

[5] A. R. Camina and C. E. Praeger, Line-transitive automorphism groups of linear spaces, Bull. London Math. Soc. 25(4) (1993), 309-315.

[6] C. J. Colbourn and M. J. Colbourn, Cyclic steiner systems having multiplier automorphisms, Util. Math. 17 (1980), 127-149.

[7] G. Cresp, Searching for line transitive, point imprimitive, linear spaces, Honours Dissertation, University of Western Australia, (available at http://arxiv.org/abs/math. C0/0604532), Perth, 2001.

[8] A. Delandtsheer and J. Doyen, Most block-transitive $t$-designs are pointprimitive, Geom. Dedicata 29(3) (1989), 307-310.

[9] Z. Janko and V. D. Tonchev, Cyclic 2-(91, 6, 1) designs with multiplier automorphisms, Discrete Math. 97(1-3) (1991), 265-268.

[10] B. D. McKay, Nauty Users Guide, Technical Report TR-CS-90-02, 1990, Computer Science Department, Australian National University.

[11] W. Nickel, A. C. Niemeyer, C. M. O’Keefe, T. Penttila and C. E. Praeger, The block-transitive, point-imprimitive 2-(729,8,1) designs, Appl. Algebra Engrg. Comm. Comput. 3(1) (1992), 47-61.

[12] C. M. O'Keefe, T. Penttila, and C. E. Praeger, Block-transitive, pointimprimitive designs with $\lambda=1$, Discrete Math. 115(1-3) (1993), 231-244. 


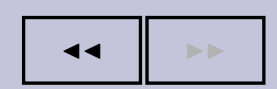

4

page $19 / 19$

go back

full screen

close

quit

[13] C. E. Praeger and N. D. Tuan, Inequalities involving the DelandtsheerDoyen parameters for finite line-transitive linear spaces, J. Combin. Theory Ser. A 102(1) (2003), 38-62.

\section{Anton Betten}

Colorado State University, Department of Mathematics, Fort Collins, Co, 80523, U.S.A. e-mail: betten@math.colostate.edu

website: http://www. math. colostate.edu/ betten

\section{Gregory Cresp}

The University of Western Australia, School of Mathematics and Statistics, 35 Stirling HighWAY, CRAWLEy 6009, Australia

e-mail: riviera@student.uwa.edu.au

Cheryl E. Praeger

The University of Western Australia, School of Mathematics and Statistics, 35 Stirling HighWAY, CRAWLEy 6009, Australia

e-mail: praeger@maths.uwa.edu.au

website: http://www.maths.uwa.edu.au/ ${ }^{\sim}$ praeger 